\title{
Performance Analysis of Edges, Corners and the genres: A Subjective Estimation
}

\author{
Mugdha Thareja ${ }^{1}$, Amit Goyal ${ }^{2}$ \\ ${ }^{I}$ (Department of Computer Science Engineering, Sri Sukhmani Institute of Engineering \& Technology, India) \\ ${ }_{2}^{2}$ (Department of Electronics and Communication Engineering, MM University-Sadopur, India)
}

\begin{abstract}
Taking a cue from its relevance in various spheres, the detection of corners and edges for an image has firmed a concrete foundation for researchers. Since the appraisal of various techniques for the same is not occluded and hence this paper brings forth the features of comparison of various techniques in order to draw difference between them. From Harris to Moravec and Gradient-based to Canny, a study as well as an objective assessment has been communicated through this article, yielding a wrap-up of features as a target for further research in this genre.
\end{abstract}

Keywords - Auto-correlation, Corner, Edge, Gradient, Image Processing.

\section{INTRODUCTION}

Digital Image Processing is a vast area of interest in exploring the advanced techniques used in interactive computer graphics and computer vision systems. It is a systematic means of processing image using computer with finite number of elements having particular location and value known as pixels [2]. Digital image processing, unlike analog, makes use of computer algorithms to perform image processing. The major advantage of digital image processing is the availability of means of avoiding noise or distortion in processing of generated signals [1]. Digital Image Processing comprises of various steps starting from acquiring an image within the problem domain to identification of desired object [23]. Before approaching to object recognition, the image is compressed and partitioned into constituent objects to derive the descriptor of the partitioned segment. This partitioning of image into its subsequent components is called the segmentation which is a binder of all the other stages of image processing [2] as depicted in fig. 1. Due to its influence on the consecutive series of functions, segmentation technology is one of the major apprehensions in digital image processing domain [17]. A highly precise partitioning brings about more successful results in object recognition. A robust method of segmentation leads to thriving results in identifying the objects independently with flourishing solutions to problems in processing the image. Feature detection is the uncovering the prominent and characteristic objects in an image. The applications of feature detection comprises of image registration, face recognition and motion tracking [3].

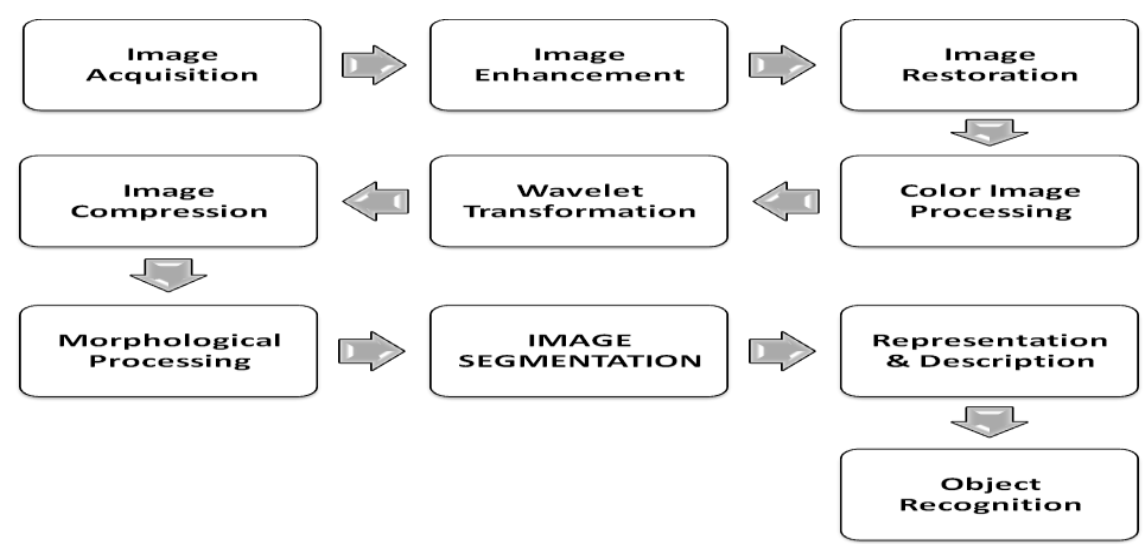

Fig.1. Catalog-Digital Image Processing [2] 


\section{CORNER DETECTION}

Corner detection is a tactic to uncover the individual, distinct feature known as corner by deducing the constituent elements of image. Corners are also known as points of interest having a defined pixel position. Corner detection includes extracting specific characteristic attributes of the constituents of image. The applications of corner detection encompass motion tracking, image combining, panorama building and 3-D graphic modeling [12]. Few of the corner detection algorithms [5, 6] are mentioned in the following sections.

Harris Corner Detector:

The Harris corner detector is insensitive to variations in scale, orientation, illumination and noise making it one of the key point detectors [9]. It works on the basis of local auto-correlation function of a signal; wherein measurement of the local changes of the signal with patches shifted by a small amount in different directions are made.

Harris points do not change with rotation. Harris detector is a region detector which gives the output descriptors taking into account the gradient difference with respect to direction, and not using the patch shift [7]. Invariance to scale is advantageous to only an extent and becomes a stone in the shoe, in case of large scale variations. The disadvantage of this detector is it is not invariant to large scale change [8]. Harris detector reveals the Ljunctions and points with the higher curvature along with the corner points for which second moment matrix and corner function is computed $[5,6]$.If both of the Eigen values of the second moment matrix are approximately equal and have high value then the taken point is detected as the corner point.

The auto-correlation function $\mathrm{C}$ as in [11] for a variation $(\partial \mathrm{x}, \partial \mathrm{y})$ through a point $(\mathrm{x}, \mathrm{y})$ of an image function represented by $\mathrm{I}(. .$, ) in the window $\mathrm{W}$ can be represented as:

$C(x, y)=\sum\left[I\left(x_{i}, y_{i}\right)-I\left(x_{i}+\partial x, y_{i}+\partial y\right)\right]^{2}$

Using First-order Taylor Expansion, the image function becomes:

$\mathrm{I}\left(\mathrm{x}_{\mathrm{i}}+\partial \mathrm{x}, \mathrm{y}_{\mathrm{i}}+\partial \mathrm{y}\right)=\mathrm{I}\left(\mathrm{x}_{\mathrm{i}}, \mathrm{y}_{\mathrm{i}}\right)+\left[\mathrm{I}_{\mathrm{x}}\left(\mathrm{x}_{\mathrm{i}}, \mathrm{y}_{\mathrm{i}}\right) \operatorname{Iy}\left(\mathrm{x}_{\mathrm{i}}, \mathrm{y}_{\mathrm{i}}\right)\right]\left[\begin{array}{c}\partial \mathrm{x} \\ \partial \mathrm{y}\end{array}\right]$

Where, $\mathrm{I}_{\mathrm{x}}(.,$.$) is partial derivative in \mathrm{x}$ and $\mathrm{I}_{\mathrm{y}}(.,$.$) is partial derivative in \mathrm{y}$. Now substituting value of equation (2) in equation (1), $\mathrm{C}(\mathrm{x}, \mathrm{y})$ becomes:

$$
\begin{aligned}
& C(x, y)=\sum_{w} I\left(x_{i}, y_{i}\right)-\left[\left(I\left(x_{i}, y_{i}\right)+I_{x}\left(x_{i}, y_{i}\right) I y\left(x_{i}, y_{i}\right)\right)\left[\begin{array}{c}
x_{x} \\
\partial y
\end{array}\right]^{2}\right. \\
& \left.=\sum_{W} I_{x}\left(x_{i}, y_{i}\right) \operatorname{Iy}\left(x_{i}, y_{i}\right)\right)\left[\begin{array}{c}
\partial x \\
\partial y
\end{array}\right]^{2} \\
& \Rightarrow \quad C(x, y)=[\partial x \partial y] \cdot c(x, y)\left[\begin{array}{c}
\partial x \\
\partial y
\end{array}\right]
\end{aligned}
$$

Here matrix $c(\mathrm{x}, \mathrm{y})$ denotes the pattern of luminous intensity of the local neighboring patches. If $\lambda_{1}$ and $\lambda_{2}$ be the Eigen values of $c(\mathrm{x}, \mathrm{y})$ for an invariant descriptor, then according to [4] it can be concluded that:

- If Eigen Value of $\lambda_{1}$ and $\lambda_{2}$ are small, then the area is depicted as flat region.

- If Eigen Value of $\lambda_{1}>\lambda_{2}$, then the area is depicted as vertical edge.

- If Eigen Value of $\lambda_{1}<\lambda_{2}$ are small, then the area depicted as horizontal edge.

- If Eigen Value of $\lambda_{1}$ and $\lambda_{2}$ are high, then the area is depicted as corner. 
In Harris-Laplace mapping, the points are detected by Harris function exactly in conformation with the scale and space selected through LoG, i.e. Laplacian- of-Gaussian operator which thus prevents the features from occurring variation in terms of direction and region chosen for scale.

\section{SUSAN Corner Detector:}

This detector [10] neither uses spatial derivatives nor does it level the image. In place of this, after applying a round mask over every pixel, the grayscale values of all the pixels coming under that mask are distinguished to that of pixel at the middle [4]. The procedure follows with calculation of the amount of pixels covered in the globular mask having almost same brightness as the centre pixel. SUSAN can thus be used for both line and edge detection [10]. SUSAN is fast, and has better repeatability rate when applied to the globular mask at different positions. The advantage of Susan over Harris is that it does not varies with rotation and change in intensity of light. However it is susceptible to noise.

\section{The Moravec Corner Detector:}

This corner detection algorithm has been the earliest methodology used to depict the corner with low similarity [4]. It proceeds with checking presence of corner if any, in every pixel in the image by defining the similarity of that pixel with neighboring and overlapping ones. This is followed by measurement of sum of squared differences (SSD) between the two patches, the similarity is deduced considering the factors as in table 1 below.

Table I Deciding Factors for Discovering Feature Points

\begin{tabular}{|c|c|}
\hline If & Then \\
\hline The sum is small & Patches are highly similar \\
\hline Illumination is uniform & Patches are considerably similar \\
\hline Pixel is found on edge & $\begin{array}{c}\text { If Perpendicular: different patches exist } \\
\text { If Parallel: look of patches has least difference }\end{array}$ \\
\hline Local Maximum & Corner Exists \\
\hline Descriptor has variations & No similarity \\
\hline
\end{tabular}

The credence of the corner to be a point of interest lies in the obtaining the smallest value of SSD between the patch and its nearby pixels in horizontal, vertical or diagonal positions. The major disadvantage of this detector lies in the obstruction that if the existing edge does not lie in the region that can be measured as nearby pixel, then the least number deduced as SSD will be large and the edge will be mistakenly taken as a feature of interest.

\section{EDGE DETECTION}

The concept of edge is based on measurement of the grayscale break-offs discovered at a point also known as gray-scale discontinuity [2]. Where the corner is meant to be two dimensional in feature, edge is formed as one dimensional object. Edge detection is a method to identify the points where intensity of brightness alters very finely. These points when clustered in line segments form edges. Edge detection distinguishes and locates discontinuities [13].

Edge detection is the most extensively applied approach towards image segmentation for the important characteristics of image like corners, lines and curves can be derived from an edge occurring in an image [18]. Thereby, the methods of edge detection are in wide applications of image partitioning and registration. These methods used are performed by doing image filtration prior to detecting edges with reduced or no noise content. The major approaches towards finding edge in an image comprise of gradient based edge detector, Sobel edge detector, and Laplacian edge detector, canny edge detector [12, 13, and 14].

\section{Gradient-based edge detector:}

It takes two dimensional derivatives while first derivative taking upon a local maxima at the edge. This is also deduced using estimated finite difference. In this, [14] the degree of gradient is represented by:

$\mathrm{g}(\mathrm{x}, \mathrm{y}) \cong\left(\partial \mathrm{x}^{2}+\partial \mathrm{y}^{2}\right)^{1 / 2}$

The angle of gradient orientation is given by the measure of $\mathrm{x}$ - axis and direction of edge at a point (x, y) is perpendicular to the direction in which gradient vector lies at that point [13].

\section{Sobel Edge Detector:}


The Sobel operator [12] performs a 2-D space matrix measurement of gradient. It finds fairly accurate magnitude of gradient at each point in a gray level image. The algorithm uses a pair of $3 \times 3$ convolution masks, one part of which approximates the gradient in the $\mathrm{x}$-direction i.e. columns while the other depicts the gradient in the $y$-direction occurring as rows. For a source image $D$, the pair of matrices $G_{x}$ and $G_{y}$ is of size $3 x 3$ containing the approximate values of derivative in the direction of $\mathrm{x}$ and $\mathrm{y}$. The scale of gradient is given by:

$|\mathrm{G}|=\sqrt{\mathrm{G}_{\mathrm{x}}}{ }^{2}+\mathrm{G}_{\mathrm{y}}^{2}$

Thus, deriving the approximation to the magnitude of gradient,

$|\mathrm{G}|=\left|\mathrm{G}_{\mathrm{x}}\right|+\left|\mathrm{G}_{\mathrm{y}}\right|$

\section{Laplacian based edge detection:}

For edge detection, the most extensively used algorithm, the Laplacian, identifies the regions of quick variations of intensity [12]. Also known as Second order derivative edge detector, this kind of maps for zero-crossings to unleash the edges. Let us consider fig. 2 for illustration, where the edge is represented by an increased intensity in signal input. Taking the first derivative or gradient of the signal it displays as follows with local maximum at the middle of the edge. If the extent of gradient surpasses a given threshold value, the location of pixel is concluded as the edge position as in Fig. 3. The succeeding derivative is of zero magnitude when the first derivative is at local maxima [14].

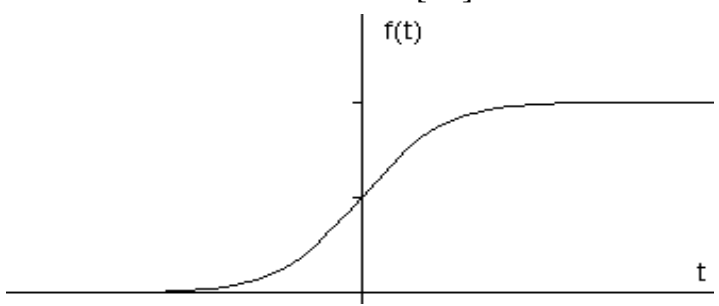

Fig. 2 input signal

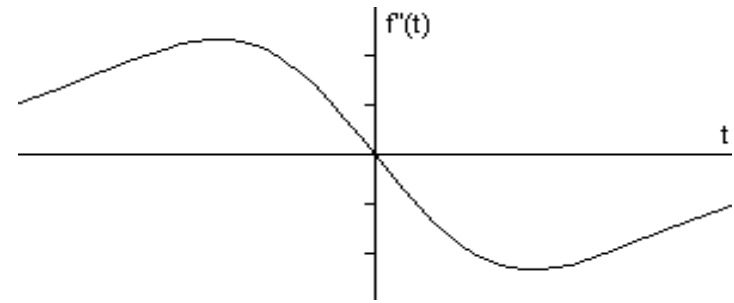

Fig. 3 derivative representation of the signal

Thus, locating the zero magnitude as in second derivative, the edge can be deduced in an image. Deciding a threshold value justifies and provides a collection of pixel values to each point of interest that works best with grayscale images with consumption of the entire range of the grayscale [14] as given below:

$$
g(x, y)= \begin{cases}1 & I(x, y)>T \\ 0 & I(x, y) \leq T\end{cases}
$$

Where $\mathrm{I}(\mathrm{x}, \mathrm{y})$ is the Image function, $\mathrm{g}(\mathrm{x}, \mathrm{y})$ is the obtained threshold image and $\mathrm{T}$ is the threshold value.

Laplacian of Gaussian:

LoG or Laplacian of Gaussian, is a 2-D or second order derivative operator used to filter the noise from the image obtained through Laplace detector [2]. The Laplacian $\mathrm{S}$ of an image function $\mathrm{m}$ at a point pixel $(\mathrm{x}, \mathrm{y})$ is given by [14]:

$\mathrm{S}(\mathrm{x}, \mathrm{y})=\nabla^{2} \mathrm{~m}(\mathrm{x}, \mathrm{y})=\frac{\partial^{2} \mathrm{~m}(\mathrm{x}, \mathrm{y})}{\partial \mathrm{x}^{2}}+\frac{\partial^{2} \mathrm{~m}(\mathrm{x}, \mathrm{y})}{\partial \mathrm{y}^{2}}$

The noise is removed to smoothen the image using an algorithm based convolution of Gaussian function in the form of square matrix of specified dimension.[14] While the Gaussian function hinders the presence of noise in image, the Laplacian operator is applied to provide zero crossing[19] edges.[22]. The LoG also known as MarrHildreth Edge Detector or the Mexican Hat operator [20] spans for unfolding the accurate location of edges and validates for broad region around the point by joining Gaussian filter with Laplacian operator to split the image with significant variation of intensity [21]. For steady intensity the output of LoG operator is null [20].

$\operatorname{LoG}(\mathrm{x}, \mathrm{y})=-1 / \pi \sigma^{4}\left(1-\left(\mathrm{x}^{2}+\mathrm{y}^{2}\right) / 2 \sigma^{2}\right) \wedge \mathrm{e}^{-(\mathrm{x} 2+\mathrm{y} 2) / 2 \sigma 2}$

The LoG responds to the occurring changes in a different way. It gives a positive response on the darker side and a negative on the lighter one. Similarly if a sharp edge occurs between regions, LoG responds with a zero 
value away from that edge; with a positive and a negative to either sides or a zero at any point on that particular edge [19].

\section{Canny Edge detector:}

Canny developed the optimal filter by increasing the resultant of the expressions of the criteria of detection and localization discussed earlier [16]. The optimal filter is estimated using first derivative of Gaussian function. It [15] transforms a window of pixels into a binary pattern based on a specified local threshold, and then applies masks to find whether an edge is present at a defined point or not.

Based on the criteria [14], the canny edge detector first levels the image to remove the noise and tracks for the image gradient to show regions with high value of derivatives in space domain. The algorithm then passes along these regions and outdoes that pixel which is not a part of maximum. The Canny edge detector [14] makes use of optimal filter based on the first derivative of a Gaussian and follows the criteria in the following stages:

- Initially, the input image is convolved with a Gaussian filter. A little blurred image is formed as compared to original. It is then improved by using Gaussian filters to remove noise contained in obtained image is before locating any edge keeping the width of filter lesser and sliding it over with the mask.

- The intensity of edge is taken out by applying gradient measurement in spatial pattern using Sobel operator followed by approximation of gradient vector.

- On the basis of the value of gradient in $\mathrm{x}$ and $\mathrm{y}$ directions of the image function, the criteria states the edge direction as $0^{\circ}$ or $90^{\circ}$. The direction of the edge is deduced using the formula as below:

$\Theta=\operatorname{intan}\left(\mathrm{G}_{\mathrm{y}} / \mathrm{G}_{\mathrm{x}}\right)$

- The break-offs falling in the depicted directions of edge are eliminated using image threshold and final image is thus formed [14].

\section{LITERATURE SURVEY}

The domain of digital image puts forward significant challenges in dealing with the problems related to computer vision system. Many of the problems in computer vision system analysis are formulated and operated on the principle of image segmentation [1]. However, many a researchers have carved out various methods to tackle with the puzzles of image segmentation, yet improvements are being made by the researchers and scholars to surpass the obstructions encountered in the algorithmic implementation of the segmentation methods including feature detection. In [3], the authors have proposed a vigorous algorithm for feature detection. In this paper, the change in local curvature and model based on scale variations is taken as base to plot the points of interest in an image for information retrieval.

Feature detection techniques involve extracting the edges and corners of the object in an image which is fairly described in [9] [15]. While edges are considered and 1-D attribute of feature, the corners are calculated by mapping onto the directions of edge been detected using the detection algorithms. The information about various corner detection techniques has been represented by Tripti Patel and S. Panchal in [4]. By studying their analysis, it is concluded that Harris and Susan relies on mask manipulation while Moravec considers the strength of corner in terms of sum of squared differences. Harris and Stephens [7] however made improvements for better result extraction.

The edge been the vital feature of an image are detected by using Sobel [12] and Canny [16] operator. The edge further can be used to derive corners and lines of the given image [13]. While the characteristic features of Harris detector have been taken into consideration for extracting corners in [4] [5] [11], SUSAN operator has been proposed to be optimal tool for low level image processing. Comparing the Harris and SUSAN detection methods, it has been come into light that Harris is better choice in corner detection than SUSAN in context to space and time complexity [13] and Laplace-Harris operator has been experimentally proven best for image enhancement in case of pre-processing of data is required [12].

\section{ASSESSMENT OF VARIOUS ALGORITHMS}

This section of the paper is dedicated to reveal the corollary of detection of corners and edges using some of the algorithms discussed above. Fig. 4 shows a 300 pixel squared grayscale image, which was taken under test in 
the MATLAB toolbox and the result of applying Harris detector to this image is shown in fig.5. The Harris operator is able to detect to the corners but it is sensitive to the very small variations in the pixel intensity and hence too many corners are detected in the image as compared to the results of Susan operator for which fig. 6 can be referred.

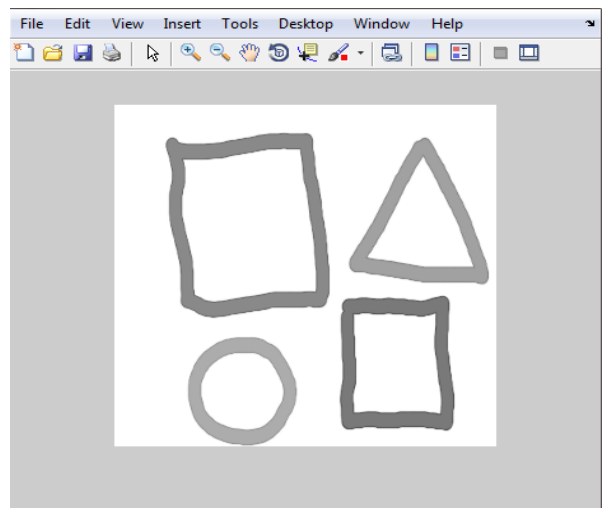

Fig.4. Test Image (300x300 Pixels, Gray Scale)

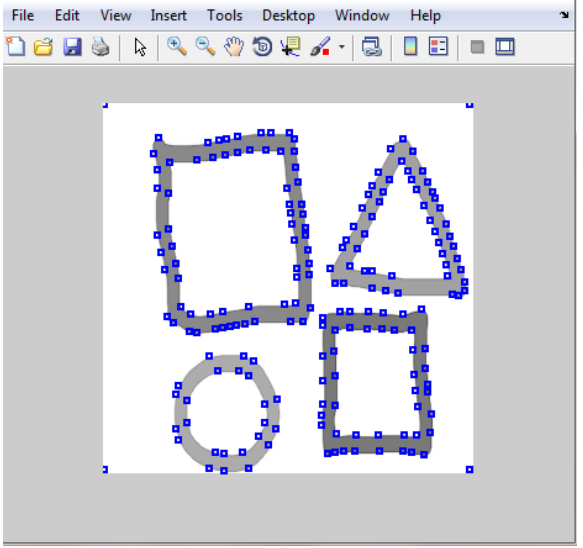

Fig.5 Detection of Corners Using Harris Operator

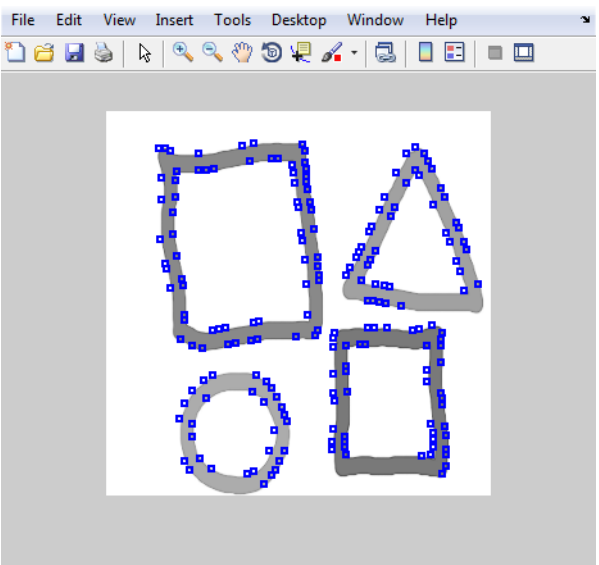

Fig.6 Detection Of Corners Using Susan Operator

To simply make a variation to the comparative study of edge detection techniques, another test image is taken; a colored image of pixel size 512x384 pixel; fig.7. Experimentation of various edge detection algorithms on this test image was done and the results of LoG and Canny edge detector are shown in fig. 8 and fig.9, respectively. In contrast to the result of LoG detector, the Canny edge detector detects more number of edges which in one way is advantageous unless the threshold for the sudden change in intensity is kept a little high which is subject to further experimentation. An edge map that reveals the contours of the object in the image is also shown in fig.

10 .

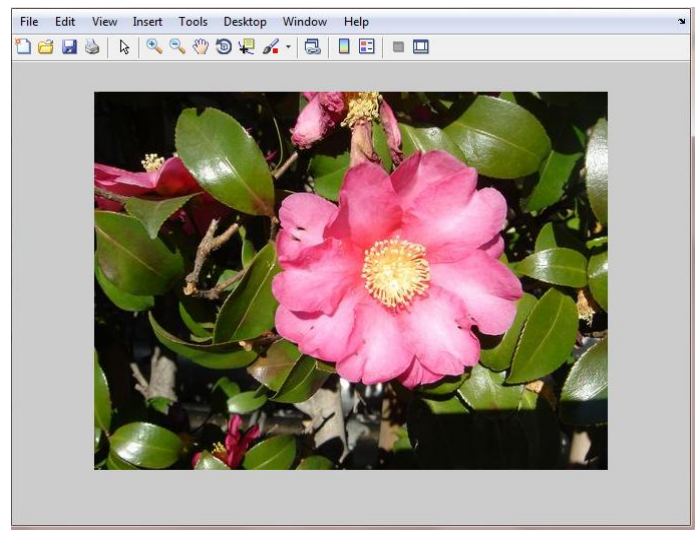

Fig.7 Test Image (512x384 Pixel)

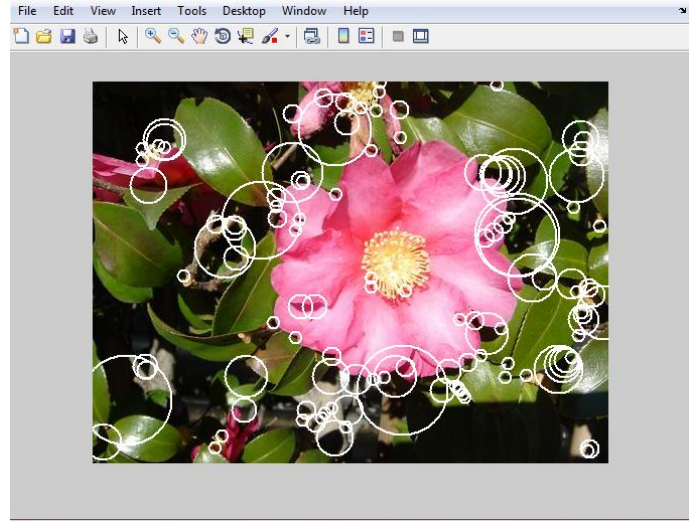

Fig.8 Edge Detection On Colored Image Using Log 


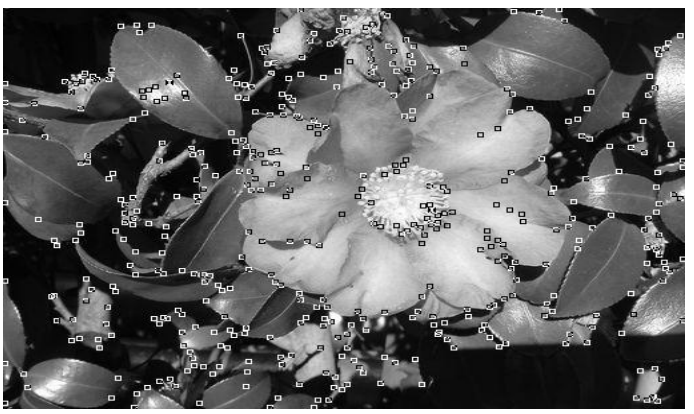

Fig.9 Edge Detection On Colored Image Using Canny

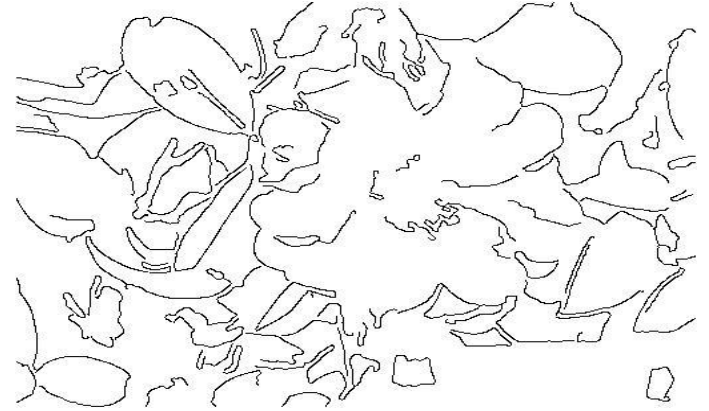

Fig.10 Edge Map Of Colored Image

\section{CONCLUSION}

The evaluation of series of experiments leads to conclude that corner detection by the Susan operator yields better results in comparison to the Harris algorithm. Analyzing the edge detection algorithms unleashes the better properties of the LoG operator. But the results of both the algorithms in their respective domains lay an emphasis on directing the research in improving them by working upon certain parameters individually in order to make these methods adaptable to all types of images.

\section{REFERENCES}

[1] A.A. Aly, S.B. Deris, and N. Zaki, Research Review for Digital Image Segmentation Techniques, International Journal of Computer Science \& Information Technology, 3(5), 2011, 99-106.

[2] R. C. Gonzalez and R. E. Woods, digital image processing 2nd ed. Prentice-Hall, 2002.

[3] B. S. Manjunath and R. Chellappa, A feature based approach to face recognition, Proc. IEEE Conf. on Computer Vision and Pattern Recognition, Champaign, IL, 1992, 373-378.

[4] T.P. Patel, and S.R. Panchal, Corner Detection Techniques: An Introductory Survey, International Journal of Engineering Development and Research, 2(4), 2014, 3680-3686.

[5] X. Gao, Z. Wenbo, F. Sattar, R. Venkateswarlu, and E. Sung, Scale-space Based Corner Detection of Gray Level Images Using Plessey Operator, Proc. $5^{\text {th }}$ IEEE International Conf. on Information, Communications and Signal Processing, Bangkok, 2005, 683687.

[6] X. Gao, Xinting, F. Sattar, and R. Venkateswarlu, Multi-Scale Corner Detection of Gray Level Images Based on Log-Gabor Wavelet Transform, IEEE Transactions on Circuits and Systems for Video Technology,17(7), 2007, 868-875.

[7] C. Harris and M. Stephens, A Combined Corner and Edge Detector, Proc. $4^{\text {th }}$ Alvey Vision Conf., Manchester, $1988,147-151$.

[8] I. Misra, S.M. Moorthi, D. Dhar and R. Ramakrishnan, An Automatic Satellite Image Registration Technique Based on Harris Corner Detection and Random Sample Consensus (RANSAC) Outlier Rejection Model, ${ }^{\text {st }}$ Intl Conf. on Recent Advances in Information Technology, RAIT, 2012, 68-73.

[9] C. Schmid, R. Mohr, and C. Bauckhage, Evaluation of Interest Point Detectors, International Journal of Computer Vision, 37(2), 2000, $151-172$.

[10] S.M. Smith, and J.M. Brady, SUSAN-A New Approach to Low Level Image Processing, International Journal of Computer Vision, 23(1), 1997, 45-78.

[11] K. G. Derpanis, the Harris Corner Detector, Proc. $4^{\text {th }}$ Alvey Vision Conf., Manchester, 2004, 1-2.

[12] N. Bhatia, and M. Chhabra, Accurate Corner Detection Methods using Two Step Approach, Global journal of Computer Science and Technology, 11(6), 2011, 25-30.

[13] S.S. Aher, Analysis of different algorithms on edge and corner detection, International Journal of Multidisciplinary Research and Development, 2(6), 2015, 99-102.

[14] M. Juneja and P.S. Sandhu, Performance Evaluation of Edge Detection Techniques for Images in Spatial Domain, International Journal of Computer Theory and Engineering, 1(5), 2009, 614-621.

[15] N. Chandrakar, and D. Bhonsle, Study and comparison of various image detection techniques, International Journal of Management, IT and Engineering, 2(5), 2012, 499-509.

[16] J. Canny, A computational approach to edge detection, IEEE Transactions on Pattern Analysis and Machine Intelligence, 8(6), 1986, 679-698.

[17] A.Y. Li, Introduction to Digital Image Pre-processing and Segmentation, $7^{\text {th }}$ International Conference on Measuring Technology and Mechatronics Automation, 2015, 588-593.

[18] S. Bhardwaj, and A. Mittal, A Survey on various Edge Detector Techniques, Procedia Technology, SciVerse ScienceDirect, Elsevier, 4, 2012, 220-226.

[19] R.M. Haralick, Zero-crossings of second directional derivative edge operator, SPIE Proc. on Robot Vision, 1982, 91-99.

[20] H. kaur, and L. Kaur, Performance Comparison of Different Feature Detection Methods with Gabor Filter, International Journal of Science and Research, 3(5), 2014, 1879-1886.

[21] P.P. Acharjya, R. Das, and D. Ghoshal, Study and Comparison of Different Edge Detectors for Image Segmentation, Global Journal of Computer Science and Technology, 12(13), 2012, 29-32.

[22] R. Kumar, and A. M. Arthanariee, International Journal of Mathematical, Computational, Physical, Electrical and Computer Engineering, 7(3), 2013, 510-514.

[23] A.Goyal, and M. Srivastava, Split and Merge based Quantitative Approach to Select Filter Combination for Image Segmentation, $4^{\text {th }}$ International Conference on Advancements in Engineering \& technology, 2016. 